\title{
Robotic dexterity: evaluation of three-dimensional monitoring system and non-dominant hand maneuverability in robotic surgery
}

\author{
Norihiko Ishikawa · Go Watanabe · Yasumitsu Hirano • \\ Noriyuki Inaki $\cdot$ Kenji Kawachi · Makoto Oda
}

Received: 29 May 2007/Accepted: 1 August 2007/Published online: 5 September 2007

(C) Springer London 2007

\begin{abstract}
There has been great progress in robotic surgical technology in recent years. The aim of this study was to objectively quantify robot-enhanced dexterity. To evaluate three-dimensional monitoring and non-dominant hand maneuverability using the da Vinci Surgical System, five surgeons were asked to thread the needle through all 11 holes on the model with handling robotic instrument. Three types of suturing were carried out. In task 1, sutures were placed using the dominant hand under 3D imaging; in task 2 , suturing was performed using the dominant hand under 2D imaging; and in task 3, suturing was done with the nondominant hand under 3D imaging. Each surgeon placed three sutures in completing each task. The time to successful completion, accuracy, and the opinion of the level of difficulty were recorded. All 45 tasks were completed. The time required to place each suture (mean $\pm \mathrm{SD}$ ) was as follows: $211.7 \pm 50.5 \mathrm{~s}$ for task $1,331.1 \pm 121.2 \mathrm{~s}$ for task 2 , and $237.1 \pm 95.7 \mathrm{~s}$ for task 3 . Task 1 took less time than task $2(P=0.02)$. There were no differences in the times between task 1 and task $3(P=0.19)$. Robotic suturing under 3D imaging is significantly faster than under $2 \mathrm{D}$ imaging, and robotic suturing using the non-dominant hand does not need significantly more time than with the non-
\end{abstract}

N. Ishikawa $(\varangle) \cdot$ Y. Hirano $\cdot$ N. Inaki

Department of Telesurgery and Geomedicine,

Kanazawa University School of Medicine, 13-1 Takara-machi,

Kanazawa 920-8641, Japan

e-mail: nori-i@mnx.jp

G. Watanabe $\cdot$ M. Oda

Department of General and Cardiothoracic Surgery, Kanazawa

University School of Medicine, Kanazawa, Japan

G. Watanabe $\cdot$ K. Kawachi

Department of Cardiac Surgery, Tokyo Medical University,

Tokyo, Japan dominant hand. Technology for robotic surgery could increase the manipulative abilities.

Keywords Robotic surgery - 3D imaging · Non-dominant hand · Dexterity

\section{Introduction}

For patients, endoscopic surgery offers the benefit of minimally invasive surgery [1]. However, for surgeons, problems include limited observation of the fixed twodimensional (2D) monitor in an unnatural posture and loss of instrumental freedom. Robotic surgical technology has allowed the disadvantages of endoscopic surgery to be overcome [2]. The da Vinci Surgical System (Intuitive Surgical Inc., Sunnyvale, CA) is a master-slave system and is more suitable than endoscopic surgery from an ergonomic perspective. The surgeon controls the da Vinci Surgical System by means of computer interface, and creates an intuitive environment under stereoscopic threedimensional (3D) imaging. Furthermore, the robotic instrument is designed so that the working tips function like a human wrist. The aim of this study was to objectively quantify the robot-enhanced dexterity.

\section{Materials and methods}

The da Vinci Surgical System was used in assessing performance of simple suturing tasks. To evaluate the da Vinci's ability to display in both a 2D and 3D mode and intuitive handling of the controller, five surgeons were asked to perform three suturing tasks using a model. 
30-cm Ti-Cron (Sherwood Davis \& Geck, MO) suture was used. Scaling was kept constant as "fine" mode throughout all tasks. The robotic instruments remained the same; the Endowist large needle driver (Intuitive Surgical Inc.) was used for tests of the dominant hand, and the Endowist deBakey forceps for the non-dominant hand, and a $0^{\circ}$ scope of ten times magnification was used on all tasks. Each surgeon had to thread the needle through all ten holes on the model by the robotic instrument (Fig. 1).

Three types of suturing task were carried out, as follows: In task 1 , by the dominant hand under 3D-imaging; in task 2 , by the dominant hand under 2D-imaging; in task 3 , by the non-dominant hand under 3D imaging. Each surgeon placed three sutures for each task. Each surgeon was permitted a 5-min practice maneuver with the robotic system on the console. In task 2, 2D imaging was obtained with a 5-mm optic camera (one side view) of the da Vinci Surgical System. To avoid bias related to the type of the task, procedures were started with task 1 followed by task 2 then task 3, and these were repeated three times each. The time to successful completion, accuracy, and the opinion of the level of difficulty were recorded.

\section{Data and statistical analysis}

Suturing times were compared using the Student's paired $t$ test. Results are expressed as mean \pm standard deviation. Differences were considered significant at a $P$ value less than 0.05 .

\section{Results}

All 45 tasks were completed. The times required for suture (mean $\pm \mathrm{SD}$ ) were as follows: task 1, $211.7 \pm 53.6 \mathrm{~s}$; task 2, $331.1 \pm 121.2 \mathrm{~s}$; and task 3,237.1 $\pm 95.2 \mathrm{~s}$. Comparing

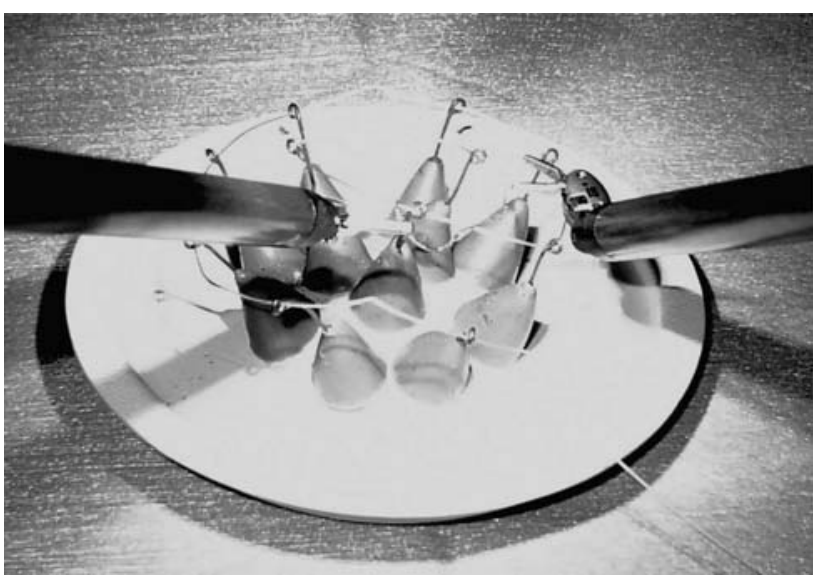

Fig. 1 Model for this study task 1 and task 2 , task 1 took slightly less time $(P=0.02)$, and robotic suturing under 3D imaging was significantly faster than under 2D imaging. Comparing task 1 and task 3, there is no difference $(P=0.19)$, and robotic suturing with the non-dominant hand does not need significantly more time than with using the non-dominant hand (Fig. 2).

\section{Discussion}

Suturing and knot-tying during endoscopic surgery cause some frustration and are time consuming. However, the surgical robot offers the advantages of allowing accurate suturing and in-depth perception during suturing. We performed this study to evaluate enhanced dexterity related to two intuitive master handles at the remote console and the 3D-imaging afforded by the da Vinci Surgical System.

We have developed a three-dimensional monitor unit that integrates the operator and the use of 3D- and 2Dmonitoring systems was evaluated. We concluded that the $3 \mathrm{D}$ monitor system was preferable to the 2D-system for advanced endoscopic surgery [3, 4]. In robotic surgery, Badani et al. [5] have reported an advantage of robotic three-dimensional suturing. The main disadvantage of the surgical robot is the lack of tactile feed back, and the console surgeon must perform suturing relying on visual feedback only. In this study, 3D imaging enables to recognize the real distance to the holes, and the surgeon could pass a surgical needle through small holes following a preindicated direction. A high-resolution, binocular, threedimensional, magnified imaging of the da Vinci Surgical System gives the console surgeon feeling of operating in an open surgical technique, and our study shows that the console surgeon can benefit from 3D imaging.

The development of robotic instruments added two more degrees of freedom than conventional endoscopic

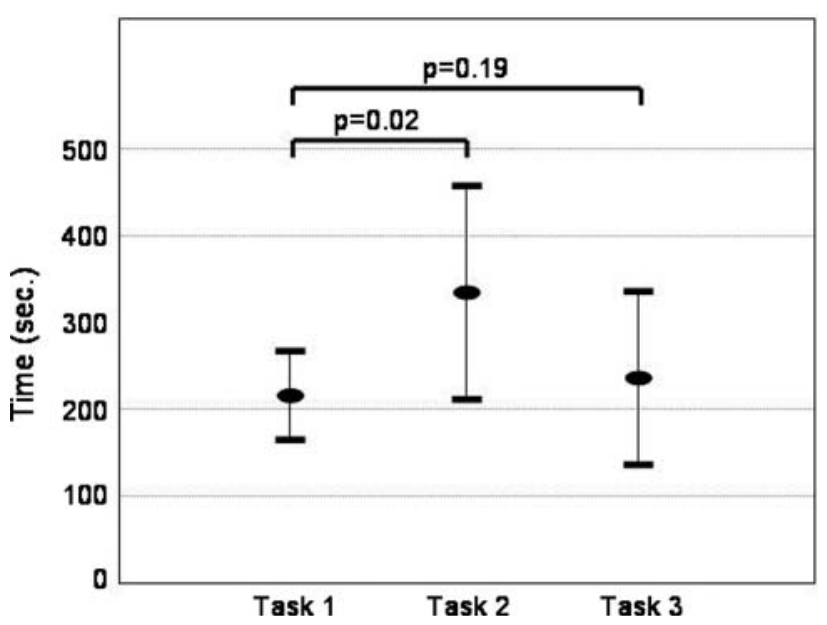

Fig. 2 Comparison of times for task 1, task 2 and task 3 
instruments to seven degrees of freedom. The presence of the "wrist-like" joint is particularly useful in procedures like suture and knot-tying, which need high maneuverability and flexibility. The da Vinci Surgical System is based on computer-assisted telemanipulation technology and computer technology, such as tremor filtration and motion scaling enhancement, which facilitate maneuverability. Moreover, not only the interface of the master console, but also finger tip controllers make wrist motion feasible and practical. We suggest that that these advantages could increase the manipulative abilities of the surgeon irrespective of the dominant or non-dominant hand. In some procedures, such as coronary artery anastomosis, the surgeon sometimes has to handle the surgical needle with the non-dominant hand, but the advanced robotic system enables the maneuver.

\section{References}

1. Williams LF Jr, Chapman WC, Bonau RA et al (1993) Comparison of laparoscopic cholecystectomy with open cholecystectomy in a single center. Am J Surg 165:459-465

2. Buess GF, Schurr MO, Fischer SC (2000) Robotics and allied technologies in endoscopic surgery. Arch Surg 135:229-235

3. Ishikawa N, Oda M, Yasumatsu H et al (2004) Three-dimensional monitor in endoscopic surgery. Surg Endosc 18:1149-1150

4. Tomita S, Watanabe G, Tabata S et al (2006) Total endoscopic beating-heart coronary artery bypass grafting using a new 3D imaging system. Innovations 1:243-246

5. Badani KK, Bhandari A, Tewari A et al (2005) Comparison of two-dimensional and three-dimensional suturing: is there a difference in a robotic surgery setting? J Endourol 19:1212-1215 